\title{
Spatiotemporal Changes to the Subventricular Zone Stem Cell Pool through Aging
}

\author{
Brett A. Shook, ${ }^{1,3}$ David H. Manz, ${ }^{1}$ John J. Peters, ${ }^{1}$ Sangwook Kang, ${ }^{2}$ and Joanne C. Conover ${ }^{1,3}$ \\ Departments of ${ }^{1}$ Physiology and Neurobiology and ${ }^{2}$ Statistics, ${ }^{3}$ Center for Regenerative Biology, University of Connecticut, Storrs, Connecticut 06269
}

Through adulthood, the rodent subventricular zone (SVZ) stem cell niche generates new olfactory bulb interneurons. We had previously reported that the number of new neurons produced in the SVZ declines through aging; however, age-related changes attributable specifically to the SVZ neural stem cell (NSC) population have not been fully characterized. Here, we conducted a spatiotemporal evaluation of adult SVZ NSCs. We assessed ventricle-contacting NSCs, which together with ependymal cells form regenerative units (pinwheels) along the lateral wall of the lateral ventricle. Based on their apical GFAP-expressing process, individual NSCs were identified across the ventricle surface using serial reconstruction of the SVZ. We observed an $86 \%$ decline in total NSCs $/ \mathrm{mm}^{2}$ of intact ependyma in 2-year old versus 3-month-old mice, with fewer NSC processes within each aged pinwheel. This resulted in an associated $78 \%$ decline in total pinwheel units $/ \mathrm{mm}^{2}$. Regional analysis along the lateral ventricle surface revealed that the age-dependent decline of NSCs and pinwheels is spatially uniform and ultimately maintains the conserved ratio of olfactory bulb interneuron subtypes generated in young mice. However, the overall neurogenic output of the aged SVZ is reduced. Surprisingly, we found no significant change in the number of actively proliferating NSCs per $\mathrm{mm}^{2}$ of ventricle surface. Instead, our data reveal that, although the total NSC number, pinwheel units and NSCs per pinwheel decline with age, the percentage of actively, mitotic NSCs increases, indicating that age-related declines in SVZmediated olfactory bulb neurogenesis occur downstream of NSC proliferation.

\section{Introduction}

In the adult mammalian brain, neurogenesis persists in a narrow region subjacent to the ependymal lining of the lateral wall of the lateral ventricles, the subventricular zone (SVZ) (GarcíaVerdugo et al., 1998; Alvarez-Buylla et al., 2001; Alvarez-Buylla and Lim, 2004). Neural stem cells (NSCs) in the SVZ have been identified as a relatively quiescent subpopulation of SVZ astrocytes (Doetsch et al., 1999; Maslov et al., 2004) that contain an apical process contacting the ventricle and a basal process contacting adjacent blood vessels (Conover et al., 2000; Mirzadeh et al., 2008; Shen et al., 2008; Tavazoie et al., 2008). This orientation is reminiscent of embryonic NSCs (radial glia), the predecessors of adult SVZ NSCs (Tramontin et al., 2003; Merkle et al., 2007; Mirzadeh et al., 2008). When viewed from the ventricle surface, ependymal cells spiral around clusters of astrocytic processes (NSCs) to form structural units termed "pinwheels" (Mirzadeh et al., 2008; Nam and Benezra, 2009). Each astrocytic process contains a primary cilium, distinguishing it from multi-ciliated ependymal cells, and is anchored at the ependymal surface via adherens and tight junctions (Doetsch et al., 1997; Mirzadeh et

Received Dec. 1, 2011; revised Feb. 27, 2012; accepted March 16, 2012.

Author contributions: B.A.S. and J.C.C. designed research; B.A.S., D.H.M., and J.J.P. performed research; B.A.S.,

S.K., and J.C.C. analyzed data; B.A.S. and J.C.C. wrote the paper.

This work was supported by National Institute of Neurological Disorders and Stroke Grant NS05033.

The authors declare no competing financial interests.

Correspondence should be addressed to Joanne C. Conover, Department of Physiology and Neurobiology, University of Connecticut, 75 North Eagleville Road, Unit 3156, Storrs, CT 06269. E-mail: joanne.conover@uconn.edu.

DOI:10.1523/JNEUROSCI.5987-11.2012

Copyright $\odot 2012$ the authors $\quad 0270-6474 / 12 / 326947-10 \$ 15.00 / 0$ al., 2008). We took advantage of these structural details to identify SVZ NSCs throughout the process of aging.

In the aged rodent brain (20-24 months old), the SVZ becomes attenuated and the total number of proliferating cells in the niche is reduced by $50-75 \%$ (Tropepe et al., 1997; Doetsch et al., 1999; Jin et al., 2003; Enwere et al., 2004; Maslov et al., 2004; Luo et al., 2006; Ahlenius et al., 2009). Similarly, thymidine analog label-retaining neuroblasts reaching the olfactory bulb (OB) are reduced by $75 \%$ in aged mice, resulting in a functional decline in fine odor discrimination (Tropepe et al., 1997; Enwere et al., 2004). Although it is well documented that neurogenesis declines with age, it is unclear how changes in the NSC pool contribute to diminished neuroblast production.

Serial reconstructions of immunostained, whole mount preparations of the lateral wall of the lateral ventricle were used to perform spatial analyses of SVZ organization, including NSC and pinwheel distribution over the course of aging. We observed stenosis and fusion (adhesion) of the lateral ventricle walls that reduced the area containing an intact ependyma and a functional SVZ niche in aged mice. Spatial analysis of regions of the ventricle surface that remained intact (no ependymal adhesion) revealed declines in ventriclecontacting NSCs and pinwheel structures and these declines were uniform across all regions. Interestingly, within these NSCcontaining regions, we observed an increase in the percentage of NSCs that were actively dividing in older mice. Together, our quantitative, spatiotemporal analyses of NSCs and pinwheel organization reveal how the SVZ stem cell niche is modified to maintain regenerative potential throughout aging. 


\section{Materials and Methods}

Animals. Male CD-1 mice were purchased from Charles River and aged in our vivarium. Three-month-old mice were designated as young adult. Six-month-old and 1-year-old mice were designated as mid-aged, and 20 - to 24-month-old mice were designated as aged. Animal procedures were performed under protocols approved by the Institutional Animal Care and Use Committee of the University of Connecticut and conform to National Institutes of Health guidelines.

Immunohistochemistry. Mice were perfused transcardially with $0.9 \%$ saline, followed by $4 \%$ paraformaldehyde (Electron Microscopy Science) in PBS. Brains were fixed overnight in $4 \%$ paraformaldehyde at $4^{\circ} \mathrm{C}$ and then washed in PBS three times (20 min each wash) before cutting into $40-50 \mu \mathrm{m}$ sections with a vibratome (VT-1000S; Leica). Tissue was then permeabilized with $0.1 \%$ Triton X-100 (Sigma) in PBS for $10 \mathrm{~min}$, blocked in $10 \%$ horse serum (Invitrogen) in PBS/1\% Triton X-100 for $1 \mathrm{~h}$, and incubated with combinations of the following primary antibodies: mouse anti-calbindin (CalB) (1:250; Sigma); goat anti-calretinin (CalR) (1:1000; Millipore Bioscience Research Reagents); goat antidoublecortin (DCX) (1:250; Santa Cruz Biotechnology); rabbit antiS100 $\beta$ (1:500; Dako); sheep anti-tyrosine hydroxylase (TH) (1:500; Abcam); and rabbit anti-TH (1:500; Pel-Freeze). After washing three times in PBS, sections were incubated with the appropriate Alexa Fluor dye-conjugated secondary antibodies (Invitrogen) for $1 \mathrm{~h}$ at room temperature or overnight at $4^{\circ} \mathrm{C}$. Secondary antibodies alone were used for controls. Sections were coverslipped with Aqua-Poly/Mount (Polysciences) and imaged on either a Carl Zeiss Axio Imager M2 microscope with Apotome (Carl Zeiss) using Hamamatsu ORCA-R ${ }^{2}$ digital camera C10600 or a Leica TCS SP2 confocal laser-scan microscope.

Whole mount dissections. The lateral wall of the lateral ventricle was dissected as described previously (Mirzadeh et al., 2008, 2010) and immunostained for the following: mouse anti- $\beta$-catenin (1:250; BD Biosciences); rabbit anti- $\beta$-catenin (1:100; Cell Signaling Technology); mouse or rabbit anti- $\boldsymbol{\gamma}$-tubulin (1:500; Sigma); mouse anti-GFAP (1:400; Millipore Bioscience Research Reagents); goat anti-GFAP (1:250; Santa Cruz Biotechnology); mouse anti-nestin (1:100; Millipore Bioscience Research Reagents); and rabbit anti-phosphohistone H3 (PH3) (1:500; Abcam). Dissected whole mounts were coverslipped with Aqua-Poly/ Mount (Polysciences) and imaged on a Leica TCS SP2 confocal laserscan microscope.

Ventricle surface area measurements. Whole mount dissections were immunostained for $\beta$-catenin and $\gamma$-tubulin and then imaged on a Leica TCS SP2 confocal laser-scan microscope. In brief, the lateral ventricle surface was visualized as a continuous sheet of ependymal cells by labeling $\beta$-catenin-expressing $\left(\beta\right.$-catenin $\left.{ }^{+}\right)$cell-cell junctions and multiple $\gamma$-tubulin ${ }^{+}$punctae, indicating basal bodies of ependymal cilia. Images were stitched together using XUVtools (www.xuvtools.org) to create a montage of the lateral wall of the lateral ventricle, converted into tiff stacks using Imaris (Bitplane), and loaded into Stereo Investigator (MicroBrightField) for additional analysis. A contour was traced around the periphery of the lateral ventricle, excluding the small posterior-most segment, as shown in the figure schematics. The area of adhesion/fusion was measured by tracing a contour around the area lacking an intact ependymal lining. By subtracting the area of adhesion from the total area of the lateral ventricle, the percentage of surface area containing a continuous ependyma was calculated. To determine age-related changes in the surface area containing intact ependyma, the percentage of surface area with intact ependyma was normalized to the percentage found in 3-month-old mice ( $n=5$ for each age).

NSC process tracking. Whole mount preparations were immunostained for $\beta$-catenin, $\gamma$-tubulin, GFAP, and PH3 or 5-ethynyl-2'deoxyuridine (EdU) (after a single intraperitoneal injection of $150 \mathrm{mg} / \mathrm{kg}$ body weight and a $2 \mathrm{~h}$ chase) and imaged on a Leica TCS SP2 confocal laser-scan microscope. Acquired $Z$-stacks were stitched together using XUVtools (www.xuvtools.org), and montages were converted into tiff stacks using Imaris (Bitplane) and loaded into Stereo Investigator (MicroBrightField) to track GFAP ${ }^{+}$NCS processes from the ventricle surface to their $\mathrm{PH}_{3}{ }^{+}$or $\mathrm{EdU}^{+}$nuclei.
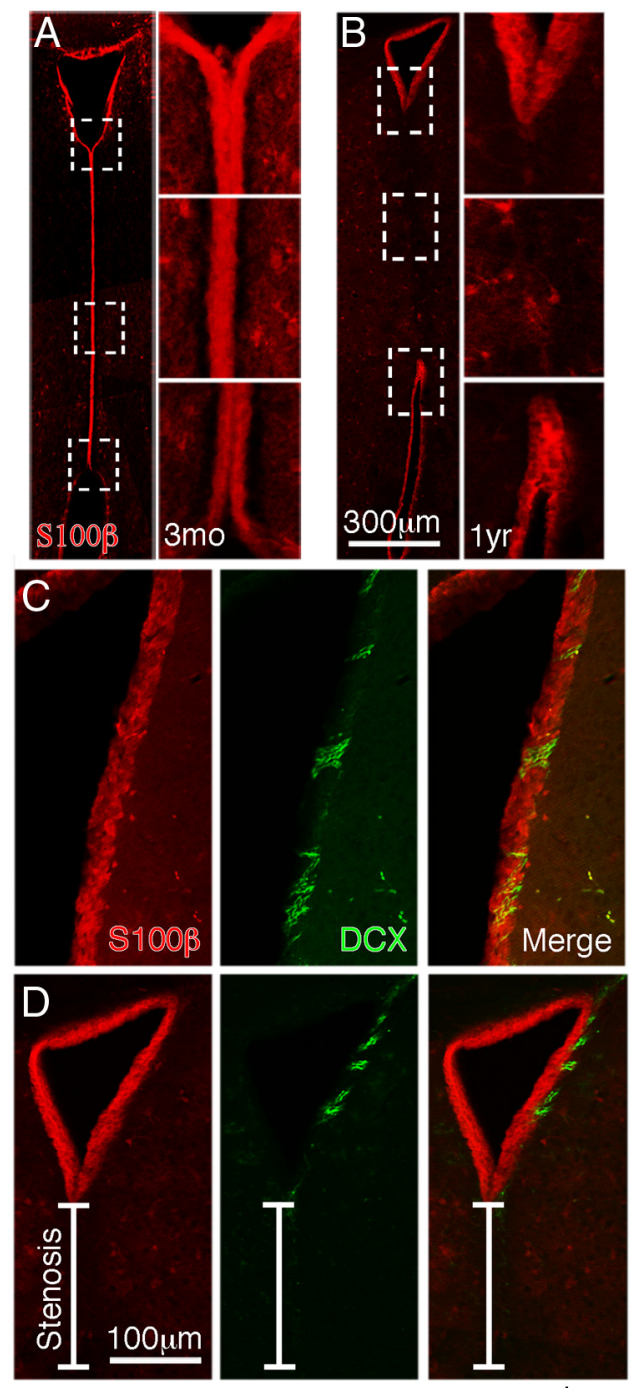

$\mathrm{E}$

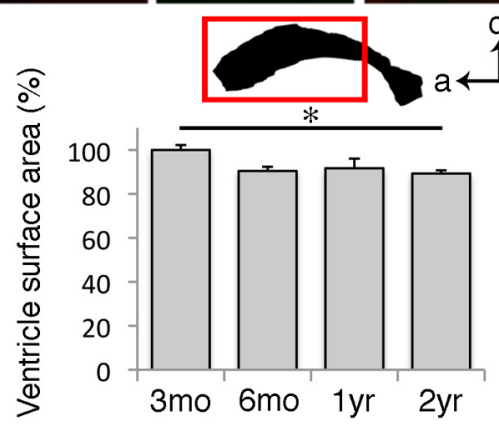

Figure 1. Ventricle wall stenosis and fusion diminishes the functional proliferative zone. $\boldsymbol{A}$, Lateral ventricle wall stenosis is clearly evident between the lateral and medial walls in the anterior lateral ventricle of young adult mice (3 months old). $\$ 100 \beta^{+}$ependymal cells are maintained at the site of adhesion. $\boldsymbol{B}$, At 1 year, ependymal cells are lost in regions of adhesion, restricting the anterior ventricle to more narrow dorsal and ventral openings (boxes indicate magnified regions). $C$, In 3-month-old mice, $D C X^{+}$neuroblasts, generated from the neurogenic SVZ, are present throughout the dorsoventral axis of the lateral wall. $\boldsymbol{D}$, In 1-year-old mice, neurogenesis and a functional SVZ are limited to areas in which an intact ependymal monolayer and CSF contact is maintained. $\boldsymbol{E}$, Using whole mount preparations, the area of adhesion was subtracted from the area of intact ependyma, and the percentage of ventricle surface area with intact ependyma was normalized to the percentage found in 3-month-old mice. This revealed a significant age-related depletion of the anterior lateral wall surface area $\left({ }^{*} p<0.05\right.$, one-way ANOVA with Bonferroni's multiple comparison post hoc test; $\left.n=5\right)$. a, Anterior; d, dorsal. 

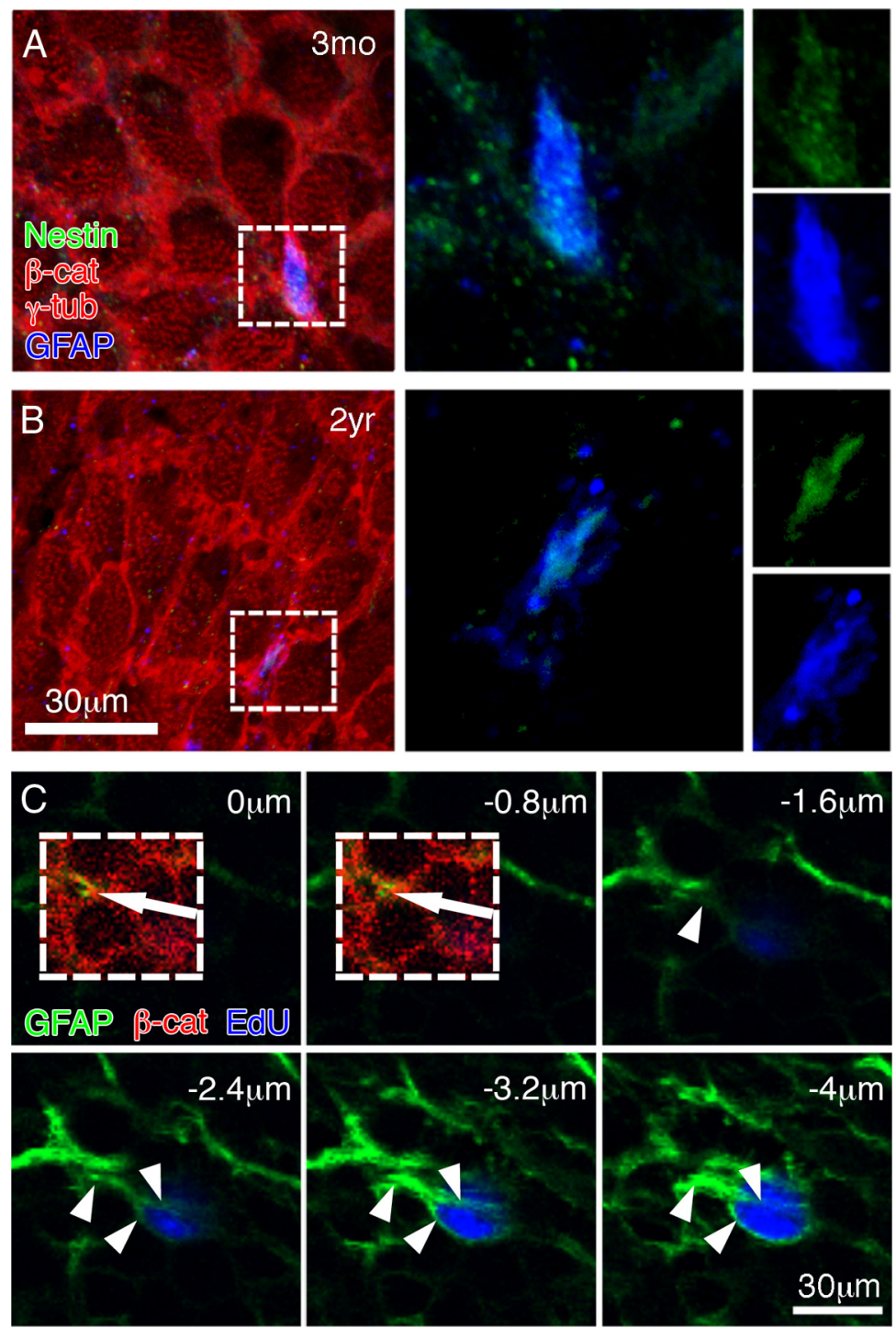

Figure 2. Ventricle-contacting astrocytes have characteristics of NSCS. $A, B$, Astrocytes contacting the lateral ventricle express the putative stem cell markers GFAP and nestin in 3-month-old $(\boldsymbol{A})$ and 2-year-old $(\boldsymbol{B})$ mice (dashed boxes indicate the magnified region). C, NSC processes can be tracked into the SVZ to identify long-term label-retaining nuclei (6-week EdU chase). $\beta$-cat, $\beta$-Catenin; $\gamma$-tub, $\gamma$-tubulin.

EdU label retention. Mice were injected intraperitoneally with three daily injections of EdU (Invitrogen) at $150 \mathrm{mg} / \mathrm{kg}$ body weight to label dividing cells. After a 6-week chase, label-retaining cells in the SVZ were examined. Tracking of NSC processes into the SVZ for identification of $\mathrm{EdU}^{+}$nuclei was performed as mentioned above. Similarly, OBs were analyzed, after a 3-week chase, to determine SVZ-generated neuroblast production. After secondary antibody incubation, EdU staining was performed per the protocol of the manufacturer. 3D montages were prepared of the OBs, using every fifth section for quantification with the virtual tissue feature of Stereo Investigator (MicroBrightField) on a Carl Zeiss Axio imager M2 microscope with Apotome (Carl Zeiss), using a Hamamatsu ORCA-R ${ }^{2}$ digital camera C10600.

Statistical analysis. Results are reported as mean \pm SEM. Statistical analysis across multiple ages was assessed using one-way ANOVA with Bonferroni's multiple comparisons post hoc test. Statistical analysis between two ages was assessed using a two-tailed unpaired Student's $t$ test. Statistical analysis conducted to assess the patterns of NSCs and pinwheels across multiple regions and ages used a general linear model for repeated measures over time. All statistical analyses were performed in Prism (GraphPad Software) or SAS 9.2 (SAS System) software. Significance was set at $p<0.05$.
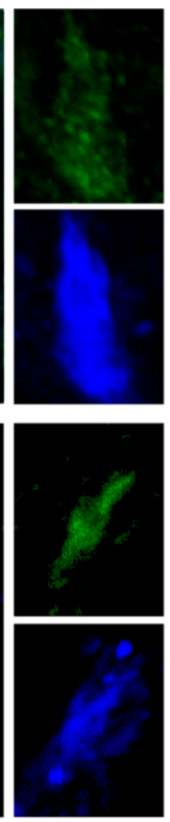

\section{Results}

Stenosis and then fusion of the lateral ventricle walls reduces the neurogenic SVZ during aging

In young (3-month-old) mice, portions of the lateral and medial anterior ventricle wall adhere to each other, but the ependymal lining remains essentially intact (lateral ventricle wall adhesion) (Fig. 1A). In contrast, in aged (20- to 24-month- old) mice, the adhered portions of the lateral and medial anterior lateral ventricle walls extend (Luo et al., 2006), a process resulting in increased lateral ventricle stenosis. By viewing ependymal cells lining the lateral ventricle with the marker $\mathrm{S} 100 \beta$, we detected deterioration and loss of the ependyma at the sites in which adhesion was observed. In 1-year-old mice, no ependymal cells remained in areas in which the lateral and medial walls had adhered and fused (Fig. $1 B)$. Neurogenesis, indicated by $\mathrm{DCX}^{+}$ neuroblasts, remained prominent in the regions of the lateral wall of the lateral ventricle containing intact ependymal cells but was absent in fused regions lacking ependymal cells (Fig. 1C,D). To determine the percentage of the lateral ventricle surface that is reduced as a result of ventricle stenosis, we prepared whole mounts of the lateral wall of the lateral ventricles and immunostained for $\beta$-catenin to label adherens junctions between ependymal cells and $\gamma$-tubulin to label the basal bodies of the cilia (Mirzadeh et al., 2008). Contiguous images were assembled into montages to reconstruct the ventricle surface. Our evaluations included the majority of the lateral wall of the lateral ventricle; only the very posterior-most region of the lateral wall, which is primarily devoid of NSCs and neuroblasts (Luskin, 1993; Doetsch and Alvarez-Buylla, 1996; Mirzadeh et al., 2008), was excluded. A continuous sheet of ependymal cells was based on observations of the $\beta$-catenin ${ }^{+}$cellular junctions of cells containing multiple clusters of $\gamma$-tubulin punctae, indicating the basal bodies of cilia. These regions were traced and used to determine the area of the intact ependymal lining along the lateral wall of the anterior lateral ventricle. Fused regions, identified by the absence of an intact ependyma (lack of a $\beta$-catenin sheet containing multiple $\gamma$-tubulin punctae), were also traced. Across the lateral wall of the lateral ventricle, the area of adhesion/fusion in 2-year-old mice reduced the surface area containing an intact ependyma to $89 \%$ of that found in 3-month-old mice (Fig. $1 E$ ). Because the SVZ and NSCs are limited to regions with an intact ependyma, this in effect depleted the area that could maintain active neurogenesis by $11 \%$ in 2 -year-old mice. Based on these results, the subsequent experiments examining SVZ NSCs throughout aging were evaluated only in regions with an intact ependyma; measurements spanned the entire ventricle wall with the exclusion of the narrow posterior-most segment (Fig. 1 E, schematic). 


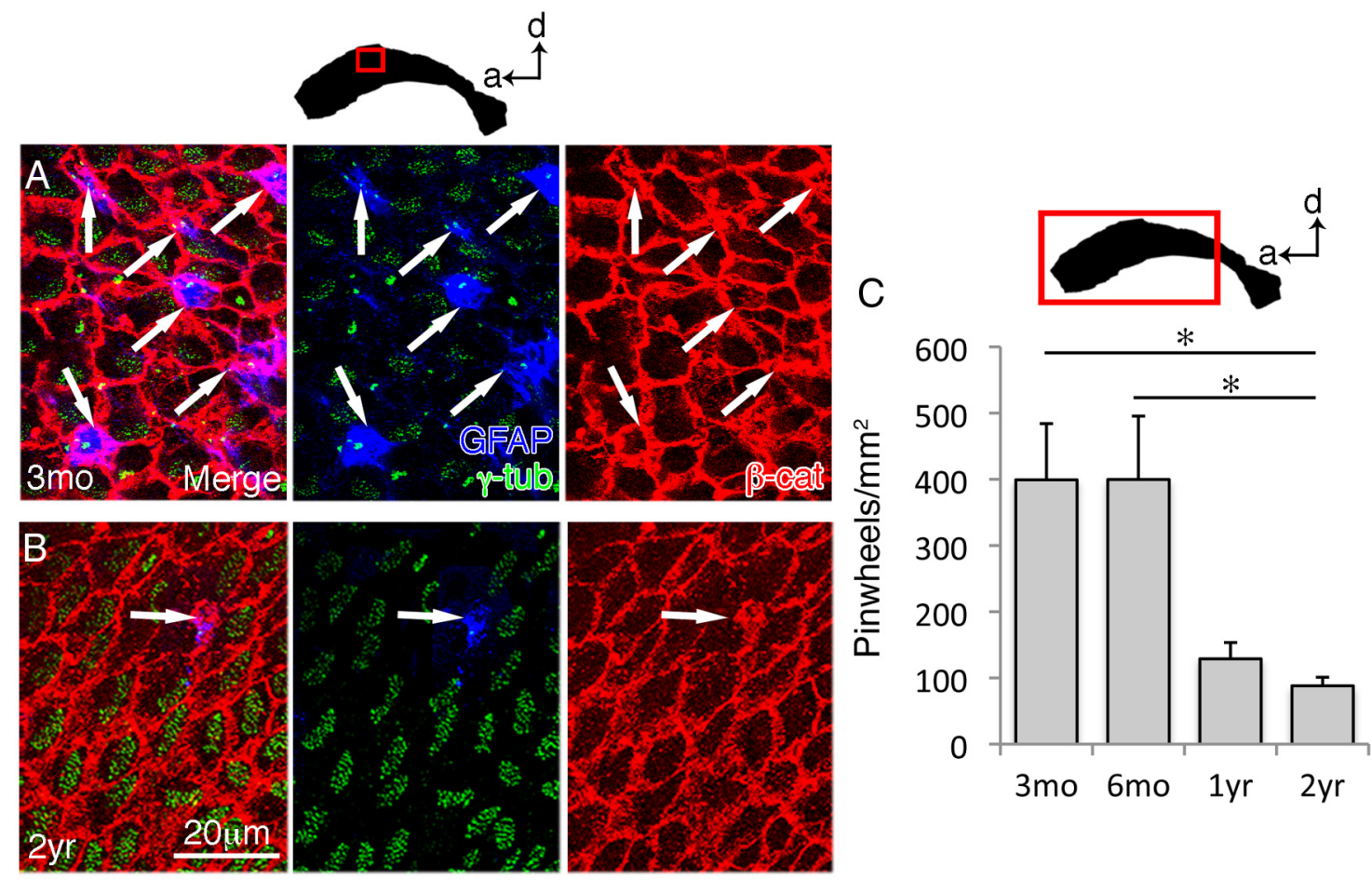

Figure 3. The number of pinwheel units at the ventricle surface decline with age. $A$, In 3-month-old mice, many pinwheel units (arrows point to astrocytic core) can be observed at the apical surface of the ventricle in whole mount preparations. $\boldsymbol{B}$, In 2-year-old mice, fewer organized pinwheels (arrow indicates single core) are observed along the ventricle surface. $\boldsymbol{C}$, With age, there is a significant decline in the number of pinwheel units at the ventricle surface $\left(n=3 ;{ }^{*} p<0.05\right.$, one-way ANOVA with Bonferroni's multiple comparison post hoc test). a, Anterior; $d$, dorsal; $\beta$-cat, $\beta$-catenin; $\gamma$-tub, $\gamma$-tubulin.

The NSC pool declines uniformly across the lateral ventricle surface with age

In the young adult, the ependymal monolayer lining the ventricle is punctuated by the apical processes of individual NSCs (Mirzadeh et al., 2008; Nam and Benezra, 2009; Kokovay et al., 2010). Astrocytes contacting the ventricle express the putative NSC markers GFAP and nestin in 3-month-old mice (Fig. 2A), as shown previously (Mirzadeh et al., 2008). Similarly, we detected GFAP ${ }^{+}$and nestin ${ }^{+}$ ventricle-contacting astrocytes in 2 -year-old mice (Fig. 2 B). To validate the identity of astrocytes with a ventricle-contacting process as NSCs, we also evaluated their ability for label-retention using the thymidine analog EdU. Three-month-old mice were given three daily injections of EdU $(150 \mathrm{mg} / \mathrm{kg})$, followed by a 6-week chase period. Only stem cells and their immediate progeny show longterm label-retention (Shen et al., 2008; Tavazoie et al., 2008). We observed that $\mathrm{GFAP}^{+}$label-retaining cells contained a $\mathrm{GFAP}^{+}$process that tracked to the ventricle surface (Fig. 2C).

To determine whether the aged ependymal wall maintains the characteristic pinwheel structures (NSC processes with encircling ependymal cells) that define the basic regenerative units of the young SVZ, we examined the lateral ventricle wall in whole mount preparations. We used $\beta$-catenin and $\gamma$-tubulin to define the ependymal cell adherens junctions and basal bodies of cilia, respectively, and GFAP to label the apical process of NSCs in 3 -month-old and 2-year-old mice. In a single field of view $(50 \times$ $65 \mu \mathrm{m})$, many pinwheels were visible at the ventricle surface in 3-month-old mice (Fig. 3A). In 2-year-old mice, NSCs and pinwheel structures were noticeably reduced across the ventricle surface (Fig. 3B). To calculate the age-related decline in the number of pinwheel structures, we analyzed only the lateral wall of the lateral ventricle containing an intact ependymal, omitting the posterior-most region of the lateral ventricle. Because all pin- wheels are lost in the region of ventricle adhesion, we examined the area of the lateral ventricle in which a continuous sheet of ependymal cells remained. After dividing the number of pinwheels by the ventricle surface area, we detected no change between the number of pinwheels $/ \mathrm{mm}^{2}$ of ventricle surface area between 3-month-old (399.22 \pm 41.47) and 6-month-old (399.74 \pm 32.48$)$ mice. However, we observed a $67 \%$ decline in pinwheel numbers in 1 -year-old mice $(128.89 \pm 11.64)$ and a $78 \%$ decline in 2-year-old mice $(88.09 \pm 11.36)$ compared with pinwheel numbers found in 3-month-old mice (Fig. $3 C$ ). These data reveal that pinwheels structures across the lateral wall of the lateral ventricle are significantly reduced with age, indicating that a key feature of the young ependymal wall is diminished in aging.

To assess changes in the number of ventricle-contacting NSCs through the process of aging, individual apical NSC processes were identified based on the presence of a single primary cilium (a single $\gamma$-tubulin ${ }^{+}$puncta compared with a cluster of $\gamma$-tubulin ${ }^{+}$puncta denoting motile cilia typical of ependymal cells) and expression of GFAP. In 3-month-old mice, pinwheel cores typically contain three or more NSC processes (Fig. 4A, C), whereas in 2-year-old mice, pinwheels contain noticeably fewer processes (Fig. 4B,C). Because the number of NSCs making up the core of the pinwheel varies between pinwheels, we examined whether pinwheels uniformly lost NSC processes by analyzing the number of NSCs within each pinwheel structure among the different age groups. With increased age, the overall number of pinwheels was reduced, and all pinwheels in older mice contained fewer NSCs per pinwheel (Fig. 4C), suggesting that NSCs are lost indiscriminately from all pinwheels. To examine the age-related decline in total NSC numbers, NSCs were quantified and normalized to the area of intact ependyma. We observed no change in the number of $\mathrm{NSCs} / \mathrm{mm}^{2}$ in 3-month-old $(993.04 \pm 98.89)$ compared with 

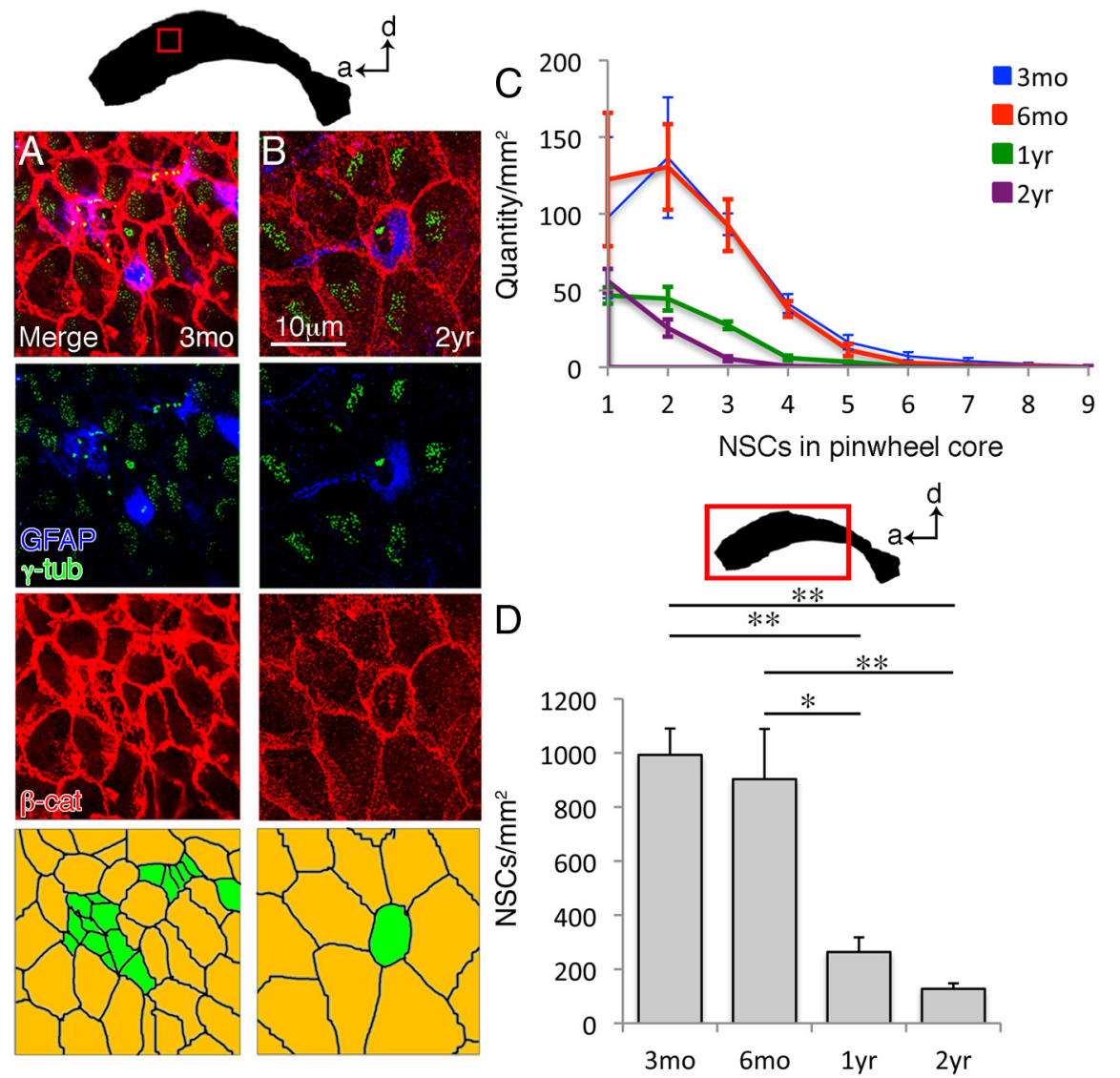

Figure 4. The total number of ventricle-contacting NSCs declines with age. A, In 3-month-old mice, pinwheel structures contain several astrocytic processes, each representing an individual NSC. The top schematic indicates the region along the lateral wall where the images were taken. The schematic under the images depicts the pinwheel cores with variable numbers of NSCs (green) surrounded by an intact ependyma (yellow). $\boldsymbol{B}$, In 2-year-old mice, most pinwheels contain only one or two NSCs (green in schematic). $\boldsymbol{C}$, With age, we observed a decline in the number of NSCs within all pinwheel cores. $\boldsymbol{D}$, The total number of NSCS contacting the anterior lateral ventricle declined with age $\left({ }^{*} p<0.05,{ }^{* *} p<0.01\right.$, one-way ANOVA with Bonferroni's multiple comparison post hoc test; $n=3$ ). a, Anterior; d, dorsal; $\beta$-cat, $\beta$-catenin; $\gamma$-tub, $\gamma$-tubulin.

6-month-old $(903.25 \pm 188.75)$ mice. However, we detected a $73 \%$ decline in 1-year-old mice $(263.51 \pm 55.11)$ and an $86 \%$ decline in 2-year-old mice $(127.11 \pm 20.94)$ compared with 3-month-old mice (Fig. 4D).

To determine whether spatial differences in NSC numbers occurred with aging, we investigated changes in pinwheel and NSC numbers regionally. Interestingly, we observed in regions in which most of the pinwheels in 3-month-old mice contained only one or two astrocytic processes in the core, these same regions in 2-year-old mice were nearly devoid of pinwheels (Fig. $5 A$ ). To determine whether the declines were spatially biased, we binned the lateral ventricle into anterior quadrants (anterior ventral, anterior dorsal, posterior ventral, and posterior dorsal) and the middle lateral ventricle. With increased age, we were able to observe a decline in both intact pinwheels and a decline in the number of NSCs in each pinwheel core across the entire lateral wall of the lateral ventricle (Fig. $5 A-D$ ). Regional analysis revealed that the age-related declines in the number of NSCs (Fig. $5 E$ ) and pinwheels (Fig. $5 F$ ) were not significantly different (NSCs, $p=0.3585$; pinwheels, $p=0.6416$ ) across all regions of the SVZ. By mapping the location of pinwheels and the number of NSCs per pinwheel core, the uniform loss of NSCs and pinwheels is revealed (Fig. 5G). It is important to note that, in the region in which ventricle wall fusion occurs, there is a complete loss of pinwheels and NSCs; however, our data indicate that where intact ependyma is found, the agerelated decline in the number of pinwheels and NSCs is spatially uniform across the lateral ventricle wall.

\section{$O B$ interneuron numbers are reduced} in aging, but the ratio of interneuron subtypes remains constant

Different regions of the SVZ differentially pattern resident stem cells for the generation of one of six unique interneuron subtypes (Hack et al., 2005; Kohwi et al., 2005; Merkle et al., 2007; Ihrie and AlvarezBuylla, 2011; Ihrie et al., 2011). We next investigated whether age-related NSC loss affects the generation of specific subpopulations of $\mathrm{OB}$ interneurons. Interneurons of the granule layer are subdivided into deep, superficial, or CalR ${ }^{+}$interneurons, whereas interneurons of the periglomerular layer are subdivided into $\mathrm{CalR}^{+}$, $\mathrm{CalB}^{+}$, or $\mathrm{TH}^{+}$interneurons (Price and Powell, 1970; Kosaka et al., 1995; Lledo et al., 2008). We predicted that a spatially uniform decline in the number of NSCs through aging would result in a similarly uniform reduction in all subpopulations of OB interneurons. The thymidine ana$\log$ EdU was administered (three daily injections of $150 \mathrm{mg} / \mathrm{kg}$ ) to label dividing cells in 3-month-old and 2-year-old mice to interrogate changes in SVZ NSC output with age. Mice were killed 3 weeks after EdU administration to allow sufficient time for newly generated neuroblasts to undergo chain migration and integrate into OB circuits (Luskin, 1993; Lois et al., 1996). Similar to previously published data (Tropepe et al., 1997; Enwere et al., 2004), we observed a $75 \%$ decline in the total number of $\mathrm{EdU}^{+}$cells reaching the olfactory bulb $(4117 \pm 615$ in 3-month-old and $1024 \pm 165$ in 2 -year-old mice) and a similar decline within the glomerular region $(598 \pm 159$ in 3-month-old and $183 \pm 31$ in 2-year-old mice) of the OB (Fig. 6A). To assess whether an age-related decline in neuroblast production differentially affected the generation of different subpopulations of interneurons, we first examined the spatial distribution of $\mathrm{EdU}^{+}$cells reaching the $\mathrm{OB}$. The number of $\mathrm{EdU}^{+}$cells reaching the glomerular region compared with the total number of $\mathrm{EdU}^{+}$cells in the $\mathrm{OB}$ remained constant with age $(14.09 \pm 1.69 \%$ in 3-month-old and $18.37 \pm$ $3.32 \%$ in 2-year-old mice). The distribution of $\mathrm{EdU}^{+}$cells in the $\mathrm{OB}$ through aging was further investigated by examining the ratio of differentiated interneuron subtypes retaining EdU within the glomeruli. EdU label-retaining, differentiated periglomerular interneurons were identified by expression of CalR, CalB, or TH (Fig. $6 B--D$ ). With increased age, the ratio of each label-retaining periglomerular subtype was maintained (Fig. $6 E$ ), as was the ratio of EdU ${ }^{+}$label-retaining cells in the glomeruli that differentiated into mature neurons (2-year-old mice, $5.68 \pm 2.04 \%$ compared with 3-month-old mice, $9.09 \pm 1.81 \%$ ). These data support the above findings that the age-related decline in SVZ neurogenic output occurs uniformly across all subpopulations of NSCs. 

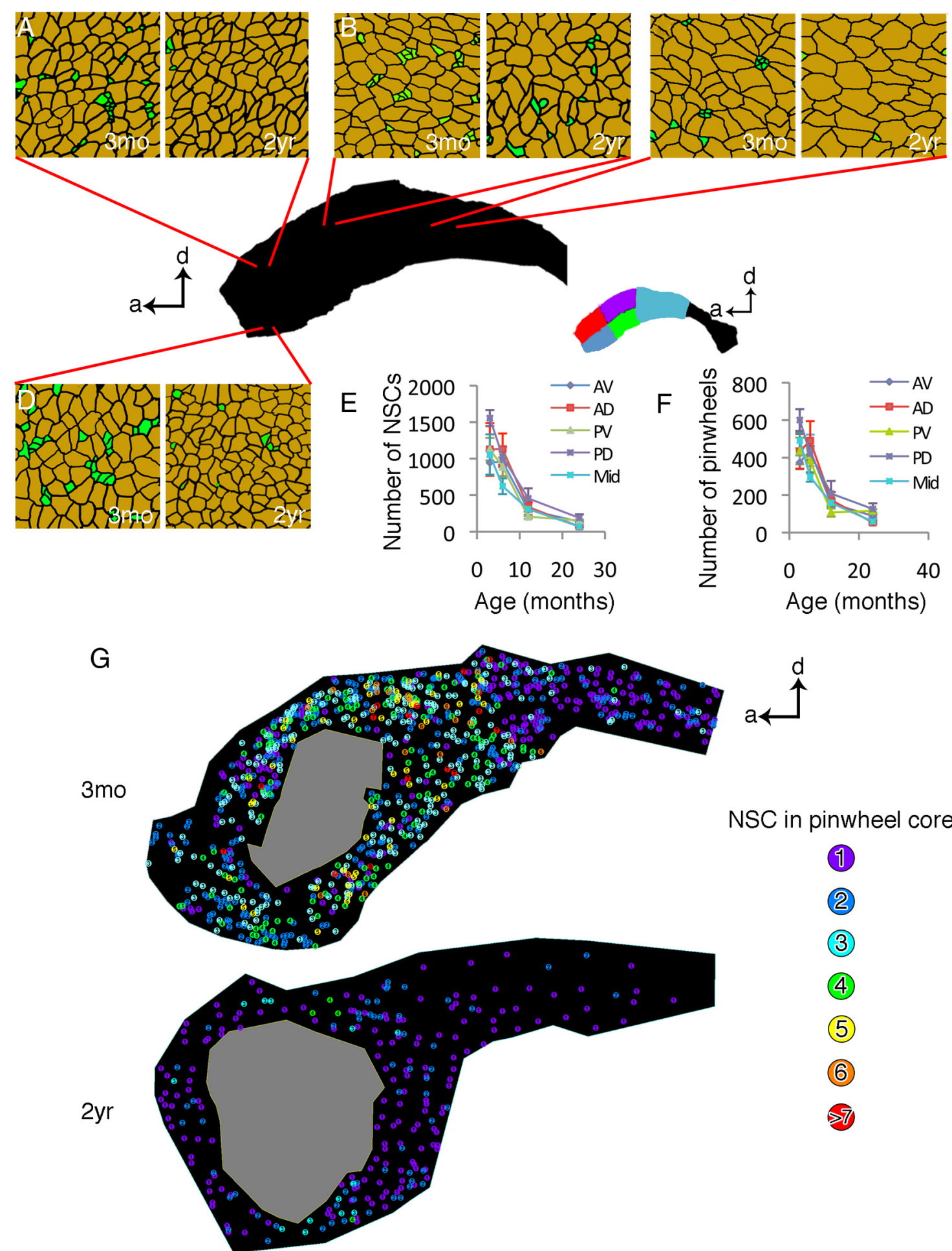

NSC in pinwheel core

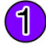

(2)

(3)

Figure 5. Throughout aging, ventricle-contacting NSCs are lost uniformly along the lateral wall of the lateral ventricles. $A-D$, Schematic representations of the ventricle surface showing NSCs (green) surrounded by ependymal cells (yellow) that were created from the original confocal images at the indicated locations along the lateral ventricle wall. An age-related decline in the number of pinwheels and a decline in the number of NSCs within the remaining pinwheel structures are clearly evident. $\boldsymbol{E}, \boldsymbol{F}$, The lateral ventricle was binned into four anterior quadrants [anterior ventral (AV), anterior dorsal (AD), posterior ventral (PV), and posterior dorsal (PD)] and the middle lateral ventricle (Mid), and pinwheel and NSCs numbers were counted in each region. No statistically significant difference in the rate of NSC (E) or pinwheel (F) loss was detected in the different regions $(n=3)$. G, The position and number of NSCs within each pinwheel core was mapped in a 3-month-old and 2-year-old mouse. A decline in the number of pinwheels and the number of NSCs within each pinwheel is observed in all regions of the map. Gray area indicates the region of adhesion/fusion. a, Anterior; d, dorsal.

An increased percentage of NSCs are proliferative in the aged SVZ

Because of limitations in identifying NSCs specifically, previous in vivo studies collectively counted all dividing cells in the SVZ for their analysis of age-related changes in the proliferative capacity of the SVZ (Tropepe et al., 1997; Enwere et al., 2004). Based on our strategy of identifying NSCs by their ventricle contact, GFAP/ nestin immunoreactivity and presence of a primary cilium, we 

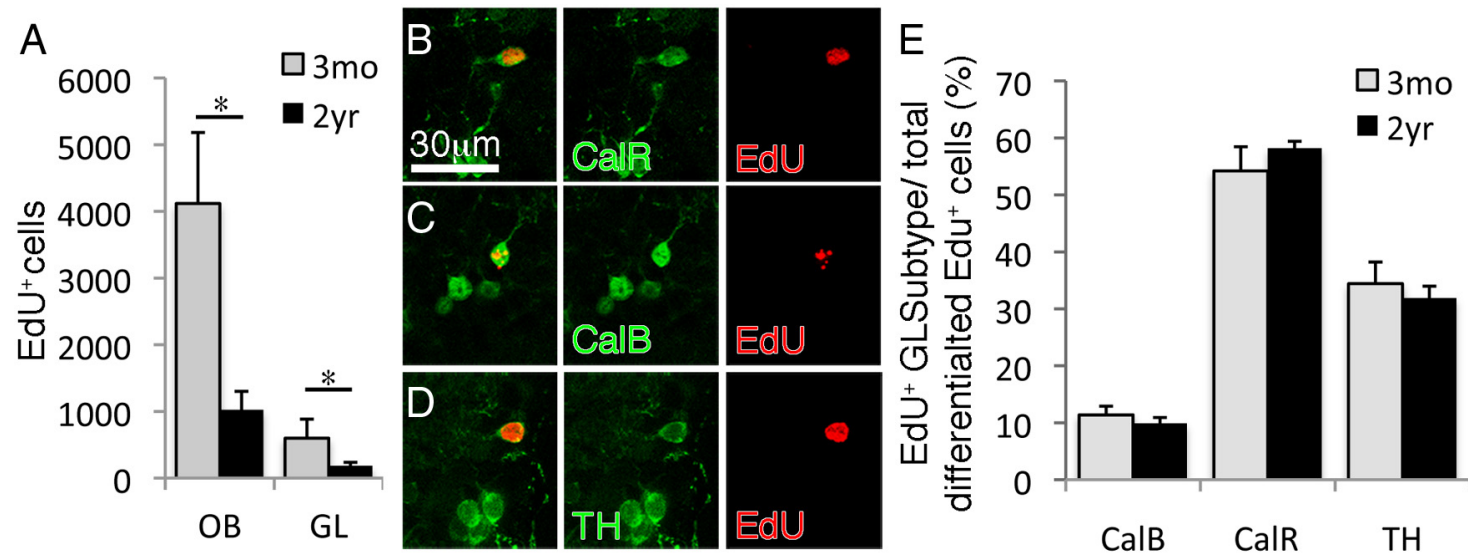

Figure 6. OB interneuron subtype ratios remain constant throughout aging. $A$, The number of EdU ${ }^{+}$cells present in the $0 B$ and within the glomerular layer is significantly decreased in 2 -year-old compared with 3-month-old mice. Periglomerular interneurons can be subdivided into $\mathrm{CaIR}^{+}(\boldsymbol{B}), \mathrm{CalB}^{+}(\boldsymbol{C})$, and $\mathrm{TH}^{+}(\boldsymbol{D})$ interneurons. $\boldsymbol{E}$, The percentage of each periglomerular interneuron subtype generated was calculated by dividing the number of $\mathrm{EdU}^{+}$cells expressing each marker by the total number of differentiated EdU ${ }^{+}$cells. No age-related change was observed in the percentage of interneuron subtypes generated $\left({ }^{*} p<0.05\right.$, Student's $t$ test). GL, Glomerular layer.

tracked the apical process of individual NSCs from the ventricular surface into the SVZ by serial reconstruction. Using the M-phase maker PH3 to detect a dividing NSC (Fig. $7 A, B$ ), we counted the number of actively proliferating NSCs. After normalization based on an intact ependymal cell layer, we observed that the number of $\mathrm{PH} 3^{+} \mathrm{NSCs} / \mathrm{mm}^{2}$ of ventricle surface area remained constant with age (Fig. $7 C$ ). When the number of mitotic NSCs $\left(\mathrm{PH}^{+}{ }^{+}\right)$was divided by the total number of NSCs, we observed an age-related increase in the percentage of NSCs undergoing mitotic division (Fig. 7D). We found that the NSC pool is more proliferative in older mice, with up to $16.89 \pm 4.73 \%$ of the NSCs undergoing mitotic division at any particular time compared with $2.34 \pm 0.21 \%$ in young mice. Together, our data show that, although the NSC pool declines with age, a greater percentage of dividing NSCs were detected in older mice ( 1 and 2 years old), perhaps to maintain comparable numbers of dividing NSCs through aging.

\section{Discussion}

Previous analyses of the aging SVZ NSC pool relied on the use of multiple thymidine analogs to evaluate global changes in SVZ proliferation or the combined use of multiple, nonspecific molecular markers to evaluate NSC numbers or in vitro neurosphere formation for analysis of proliferative potential (Tropepe et al., 1997; Enwere et al., 2004; Maslov et al., 2004). Results from these studies have ranged from no detectable change to an $80 \%$ decline in the NSC pool in 2-year-old compared with 3-month-old mice. The unique cytoarchitecture of the SVZ at the ventricle surface (Mirzadeh et al., 2008) permits a distinctly different approach to assess NSC changes, and our study represents the first examination of the SVZ NSC population that uses both molecular markers and cytoarchitectural attributes to quantify NSCs throughout the process of aging. Using this method, we report an $86 \%$ decline in the number of ventricle-contacting NSCs, resulting in a 78\% loss of pinwheel structures in 2-year-old mice compared with 3-month-old mice. This decline is uniform across regions of the lateral wall that maintain an intact ependymal cell lining. However, it should be noted that the age-related decline in the total number of ventricle-contacting NSCs is further exacerbated by an $11 \%$ loss of anterior lateral ventricle surface area as a result of fusion of the lateral ventricle walls and loss of the underlying SVZ.

Currently, the fate of the NSCs that are removed from the stem cell pool is unknown. Recently, it was shown that hippocampal NSCs transition into non-stem cell astrocytes once they have gone through several rounds of division (Encinas et al., 2011). This suggests that adult stem cells do not have unlimited self-renewal potential. Given that the number of astrocytes within the SVZ does not change with age (Luo et al., 2006), the generation of non-stem cell astrocytes in the SVZ by NSCs exiting the stem cell pool may occur but would appear to be limited. Our previous work demonstrated that proliferative astrocytes of the SVZ intercalate within the ependymal lining after ependymal cell loss, contributing to maintenance of the ependymal barrier (Luo et al., 2006, 2008). Over time, some of these intercalated astrocytes acquire morphological and antigenic characteristics of mature ependymal cells (Luo et al., 2008). Because NSCs contact the ventricle, are proliferative, and have tight and adherens junctions with neighboring ependymal cells, they are the most logical source of proliferative SVZ astrocytes to function in this form of SVZ-mediated regenerative repair. Although it is currently unclear whether ependymal wall repair requires NSCs to exit the stem cell pool (e.g., both cells incorporate within the ependymal lining as replacement cells), if they do, this would be a mechanism that could help explain the age-related declines in NSC numbers.

Similar to previous results (Enwere et al., 2004; Molofsky et al., 2006), we detected a $75 \%$ decline in the number of neuroblasts reaching the OB 3 weeks after administration of EdU in 2 -year-old mice. Here, we demonstrate that, corresponding to a spatially uniform decline in NSCs, the distribution of EdU ${ }^{+}$ cells within the $\mathrm{OB}$ remained constant with age, and an agedependent decline of newly generated interneurons occurred equally for each interneuron subtype. Similarly, in the transition from early neonate to young adult mouse, the ratio of interneuron subtypes generated remained constant unless there was injury or input deprivation through naris closure (Bovetti et al., 2007; De Marchis et al., 2007; Batista-Brito et al., 2008; Sawada et al., 2011). Therefore, the maintenance of $\mathrm{OB}$ interneuron subtype production throughout aging is consistent with the uniform decline in NSCs in different regions of the SVZ. It is possible that the conserved decline among each 
interneuron subtype results from events that occur while neuroblasts are migrating from the SVZ to the OB. These events may include selective cell death of specific interneuron subtypes or changes in migration pathways leading to support of particular interneuron subtypes and may not be based solely on the NSC populations along the lateral ventricle wall. Although fusion of the lateral ventricle walls reduces the SVZ by $11 \%$, this loss does not appear to have a detectable effect on a specific subtype of $\mathrm{OB}$ interneuron. Pinwheels within the area of stenosis progression contain fewer NSCs than pinwheels that are unaffected by ventricular stenosis (Mirzadeh et al., 2008), most likely limiting their impact on $\mathrm{OB}$ interneuron production.

According to SVZ regeneration studies (Doetsch et al., 1999), NSC division generates an immature highly proliferative progenitor cell (type- $\mathrm{C}$ cell) that can undergo several divisions and subsequently divide to generate proliferative neuroblasts. Our data demonstrate that the agerelated decline in neurogenesis is not attributable to a change in the number of actively proliferating NSCs; rather, both type- $\mathrm{C}$ cells and neuroblasts decline, as reported previously. Because the NSC pool is dramatically reduced in aging, sustaining the same number of active NSCs would require that a greater percentage of NSCs are actively proliferating in the aged SVZ, as our data demonstrate. Given the recent finding that the cell cycle rate of NSCs does not change with age (Stoll et al., 2011), the percentage of NSCs that are actively proliferating must thereby increase with age. In vitro and in vivo analysis also demonstrated that proliferative cells immunoreactive for putative NSC markers showed increased reentry into the cell cycle in aged mice compared with young adult mice (Stoll et al., 2011). These data support our finding that a greater percentage of the ventricle-contacting NSC pool of cells is mitotically active in aged mice.

Because a proliferative NSC population is maintained with increased age, the decline in SVZ neurogenesis must be attributable to factors acting downstream of NSC activation. In other stem cell niches, aged spermatogonial stem cells can be serially transplanted into young hosts without a decline in function for years (Ryu et al., 2006), and muscle satellite cells continue to function properly in aged mice when paired to the systemic factors of young mice through parabiosis (Conboy et al., 2005). Jin et al. (2003) were able to demonstrate that, after infusions of FGF or EGF, the level of total proliferation in the aged SVZ was comparable with the young adult, and aged NSCs isolated using a nestin-GFP mouse can generate the same number of neurospheres as young NSCs (Ahlenius et al.,

C
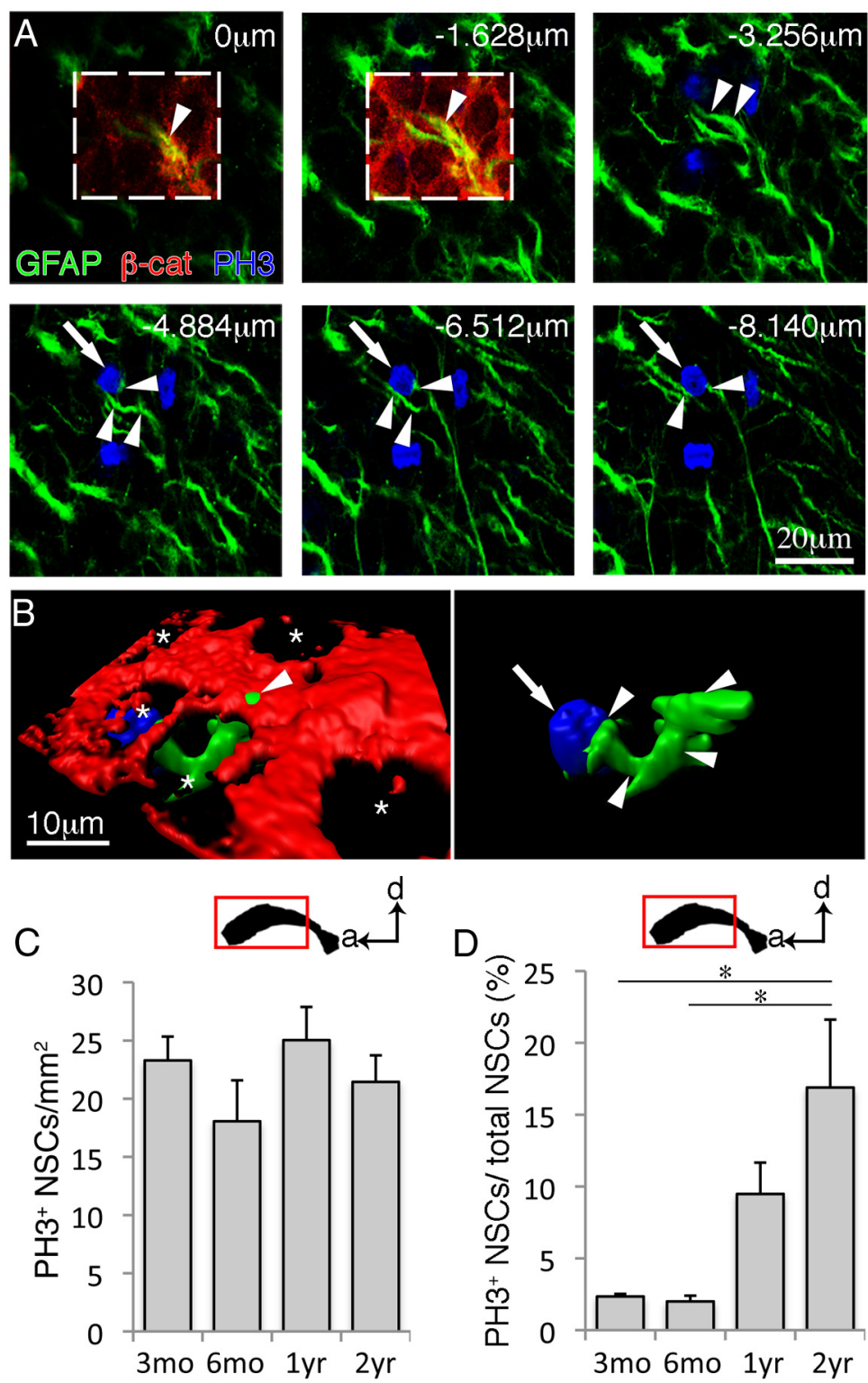

Figure 7. The percentage of mitotic NSC increases with age. $\boldsymbol{A}$, The apical process of an NSC (arrowheads) can be visualized at the ventricular surface and followed down to its nuclei (arrow) as a means to identify mitotic NSCs. $\boldsymbol{B}$, Imaris reconstruction of the $\beta$-Catenin (red) outlines ependymal cells, and the asterisks denote the cytoplasm of ependymal cells. $C$, Using this method to detect mitotic NSCs, the number of $\mathrm{PH} 3{ }^{+} \mathrm{NSCs} / \mathrm{mm}^{2}$ of ventricle surface area remained constant through aging. $\boldsymbol{D}$, When the number of $\mathrm{PH}^{+}{ }^{+} \mathrm{NSCs}$ is divided by the number of existing NSCs, there is a significant increase in the percentage of mitotically active NSCs $\left({ }^{*} p<0.05\right.$, one-way ANOVA with Bonferroni's multiple comparison post hoc test). a, Anterior; d, dorsal.

2009). These data suggest that aged stem cells maintain the same proliferative potential as young adult mice, and a decline in available growth factors or other niche-associated factors likely contribute to the diminished neurogenic niche. Thus, the loss in neurogenic capacity appears to stem from defects found in type-C cells and neuroblasts and not NSCs. It remains to be determined how the aged SVZ increases NSC activation. One possibility is the unique organization of the fractone network within the SVZ. This extension of the basal lamina from subjacent blood vessels abuts the basal membrane of the ependymal cell layer and can aid in sequestering growth factors necessary for SVZ proliferation (Mercier et al., 2002; Kerever et al., 2007). This network may preferentially distribute growth factors to NSCs based on its physical prox- 
imity to the ependyma. Alternatively, a negative feedback loop between NSCs and their immediate progeny, type-C progenitor cells, has been shown to inhibit NSC proliferation (Aguirre et al., 2010). This feedback loop, functioning through the interaction of Notch and EGF receptor pathways, has been shown to maintain the balance between NSC and progeny numbers in the young adult mouse. Based on the reduced number of type-C cells in the aged mouse (Luo et al., 2006), increased NSC activation may result. Additional investigation is required to determine the mechanism controlling increased NSC activation in the aged SVZ.

In summary, our results show that, through the process of aging, the highly proliferative SVZ becomes restricted as a result of loss from ventricular stenosis and adhesion. With increased age, total NSC numbers decline in a spatially uniform manner across the remaining ventricle surface. This results in a uniform loss of organized pinwheel structures, with regional subpopulations of NSCs equally affected. As a consequence, the aged SVZ generates the same ratio of $\mathrm{OB}$ interneurons, albeit at reduced numbers. Importantly, we found that the aged SVZ maintains the same number of mitotically active NSCs as the young adult. When dividing NSCs are normalized to the total number of NSCs, an age-related increase in the percentage of proliferative NSCs is observed-with increased age, more NSCs are actively dividing. This study provides critical information about how SVZ niche dynamics change through aging and helps reconcile existing data on age-related changes to the SVZ and decreased SVZ neurogenic capacity.

\section{References}

Aguirre A, Rubio ME, Gallo V (2010) Notch and EGFR pathway interaction regulates neural stem cell number and self-renewal. Nature 467:323-327.

Ahlenius H, Visan V, Kokaia M, Lindvall O, Kokaia Z (2009) Neural stem and progenitor cells retain their potential for proliferation and differentiation into functional neurons despite lower number in aged brain. J Neurosci 29:4408-4419.

Alvarez-Buylla A, Lim DA (2004) For the long run: maintaining germinal niches in the adult brain. Neuron 41:683-686.

Alvarez-Buylla A, García-Verdugo JM, Tramontin AD (2001) A unified hypothesis on the lineage of neural stem cells. Nat Rev Neurosci 2:287-293.

Batista-Brito R, Close J, Machold R, Fishell G (2008) The distinct temporal origins of olfactory bulb interneuron subtypes. J Neurosci 28:3966-3975.

Bovetti S, Peretto P, Fasolo A, De Marchis S (2007) Spatio-temporal specification of olfactory bulb interneurons. J Mol Histol 38:563-569.

Conboy IM, Conboy MJ, Wagers AJ, Girma ER, Weissman IL, Rando TA (2005) Rejuvenation of aged progenitor cells by exposure to a young systemic environment. Nature 433:760-764.

Conover JC, Doetsch F, Garcia-Verdugo JM, Gale NW, Yancopoulos GD, Alvarez-Buylla A (2000) Disruption of Eph/ephrin signaling affects migration and proliferation in the adult subventricular zone. Nat Neurosci 3:1091-1097.

De Marchis S, Bovetti S, Carletti B, Hsieh YC, Garzotto D, Peretto P, Fasolo A, Puche AC, Rossi F (2007) Generation of distinct types of periglomerular olfactory bulb interneurons during development and in adult mice: implication for intrinsic properties of the subventricular zone progenitor population. J Neurosci 27:657-664.

Doetsch F, Alvarez-Buylla A (1996) Network of tangential pathways for neuronal migration in adult mammalian brain. Proc Natl Acad Sci U S A 93:14895-14900.

Doetsch F, García-Verdugo JM, Alvarez-Buylla A (1997) Cellular composition and three-dimensional organization of the subventricular germinal zone in the adult mammalian brain. J Neurosci 17:5046-5061.

Doetsch F, Caill é I, Lim DA, García-Verdugo JM, Alvarez-Buylla A (1999) Subventricular zone astrocytes are neural stem cells in the adult mammalian brain. Cell 97:703-716.

Encinas JM, Michurina TV, Peunova N, Park JH, Tordo J, Peterson DA,
Fishell G, Koulakov A, Enikolopov G (2011) Division-coupled astrocytic differentiation and age-related depletion of neural stem cells in the adult hippocampus. Cell Stem Cell 8:566-579.

Enwere E, Shingo T, Gregg C, Fujikawa H, Ohta S, Weiss S (2004) Aging results in reduced epidermal growth factor receptor signaling, diminished olfactory neurogenesis, and deficits in fine olfactory discrimination. J Neurosci 24:8354-8365.

García-Verdugo JM, Doetsch F, Wichterle H, Lim DA, Alvarez-Buylla A (1998) Architecture and cell types of the adult subventricular zone: in search of the stem cells. J Neurobiol 36:234-248.

Hack MA, Saghatelyan A, de Chevigny A, Pfeifer A, Ashery-Padan R, Lledo PM, Götz M (2005) Neuronal fate determinants of adult olfactory bulb neurogenesis. Nat Neurosci 8:865-872.

Ihrie RA, Alvarez-Buylla A (2011) Lake-front property: a unique germinal niche by the lateral ventricles of the adult brain. Neuron 70:674-686.

Ihrie RA, Shah JK, Harwell CC, Levine JH, Guinto CD, Lezameta M, Kriegstein AR, Alvarez-Buylla A (2011) Persistent sonic hedgehog signaling in adult brain determines neural stem cell positional identity. Neuron 71:250-262.

Jin K, Sun Y, Xie L, Batteur S, Mao XO, Smelick C, Logvinova A, Greenberg DA (2003) Neurogenesis and aging: FGF-2 and HB-EGF restore neurogenesis in hippocampus and subventricular zone of aged mice. Aging Cell 2:175-183.

Kerever A, Schnack J, Vellinga D, Ichikawa N, Moon C, Arikawa-Hirasawa E, Efird JT, Mercier F (2007) Novel extracellular matrix structures in the neural stem cell niche capture the neurogenic factor fibroblast growth factor 2 from the extracellular milieu. Stem Cells 25:2146-2157.

Kohwi M, Osumi N, Rubenstein JL, Alvarez-Buylla A (2005) Pax6 is required for making specific subpopulations of granule and periglomerular neurons in the olfactory bulb. J Neurosci 25:6997-7003.

Kokovay E, Goderie S, Wang Y, Lotz S, Lin G, Sun Y, Roysam B, Shen Q, Temple S (2010) Adult SVZ lineage cells home to and leave the vascular niche via differential responses to SDF1/CXCR4 signaling. Cell Stem Cell 7:163-173.

Kosaka K, Aika Y, Toida K, Heizmann CW, Hunziker W, Jacobowitz DM, Nagatsu I, Streit P, Visser TJ, Kosaka T (1995) Chemically defined neuron groups and their subpopulations in the glomerular layer of the rat main olfactory bulb. Neurosci Res 23:73-88.

Lledo PM, Merkle FT, Alvarez-Buylla A (2008) Origin and function of olfactory bulb interneuron diversity. Trends Neurosci 31:392-400.

Lois C, García-Verdugo JM, Alvarez-Buylla A (1996) Chain migration of neuronal precursors. Science 271:978-981.

Luo J, Daniels SB, Lennington JB, Notti RQ, Conover JC (2006) The aging neurogenic subventricular zone. Aging Cell 5:139-152.

Luo J, Shook BA, Daniels SB, Conover JC (2008) Subventricular zonemediated ependyma repair in the adult mammalian brain. J Neurosci 28:3804-3813.

Luskin MB (1993) Restricted proliferation and migration of postnatally generated neurons derived from the forebrain subventricular zone. Neuron 11:173-189.

Maslov AY, Barone TA, Plunkett RJ, Pruitt SC (2004) Neural stem cell detection, characterization, and age-related changes in the subventricular zone of mice. J Neurosci 24:1726-1733.

Mercier F, Kitasako JT, Hatton GI (2002) Anatomy of the brain neurogenic zones revisited: fractones and the fibroblast/macrophage network. J Comp Neurol 451:170-188.

Merkle FT, Mirzadeh Z, Alvarez-Buylla A (2007) Mosaic organization of neural stem cells in the adult brain. Science 317:381-384.

Mirzadeh Z, Merkle FT, Soriano-Navarro M, Garcia-Verdugo JM, AlvarezBuylla A (2008) Neural stem cells confer unique pinwheel architecture to the ventricular surface in neurogenic regions of the adult brain. Cell Stem Cell 3:265-278.

Mirzadeh Z, Doetsch F, Sawamoto K, Wichterle H, Alvarez-Buylla A (2010) The subventricular zone en-face: wholemount staining and ependymal flow. J Vis Exp pii:1938.

Molofsky AV, Slutsky SG, Joseph NM, He S, Pardal R, Krishnamurthy J, Sharpless NE, Morrison SJ (2006) Increasing p16INK4a expression decreases forebrain progenitors and neurogenesis during ageing. Nature 443:448-452.

Nam HS, Benezra R (2009) High levels of Id1 expression define B1 type adult neural stem cells. Cell Stem Cell 5:515-526. 
Price JL, Powell TP (1970) The synaptology of the granule cells of the olfactory bulb. J Cell Sci 7:125-155.

Ryu BY, Orwig KE, Oatley JM, Avarbock MR, Brinster RL (2006) Effects of aging and niche microenvironment on spermatogonial stem cell selfrenewal. Stem Cells 24:1505-1511.

Sawada M, Kaneko N, Inada H, Wake H, Kato Y, Yanagawa Y, Kobayashi K, Nemoto T, Nabekura J, Sawamoto K (2011) Sensory input regulates spatial and subtype-specific patterns of neuronal turnover in the adult olfactory bulb. J Neurosci 31:11587-11596.

Shen Q, Wang Y, Kokovay E, Lin G, Chuang SM, Goderie SK, Roysam B, Temple S (2008) Adult SVZ stem cells lie in a vascular niche: a quantitative analysis of niche cell-cell interactions. Cell Stem Cell 3:289-300.

Stoll EA, Habibi BA, Mikheev AM, Lasiene J, Massey SC, Swanson KR, Ros- tomily RC, Horner PJ (2011) Increased re-entry into cell cycle mitigates age-related neurogenic decline in the murine subventricular zone. Stem Cells 29:2005-2017.

Tavazoie M, Van der Veken L, Silva-Vargas V, Louissaint M, Colonna L, Zaidi B, Garcia-Verdugo JM, Doetsch F (2008) A specialized vascular niche for adult neural stem cells. Cell Stem Cell 3:279-288.

Tramontin AD, García-Verdugo JM, Lim DA, Alvarez-Buylla A (2003) Postnatal development of radial glia and the ventricular zone (VZ): a continuum of the neural stem cell compartment. Cereb Cortex 13:580-587.

Tropepe V, Craig CG, Morshead CM, van der Kooy D (1997) Transforming growth factor-alpha null and senescent mice show decreased neural progenitor cell proliferation in the forebrain subependyma. J Neurosci 17: $7850-7859$. 